http://doi.org/10.15359/ree.13-2.3

\title{
Validación del software educativo Poliestudio 1.0: informe de investigación ${ }^{1}$
}

\author{
Juan José Fallas M. ${ }^{2}$ \\ Instituto Tecnológico de Costa Rica, Escuela de Matemática, \\ Cartago, Costa Rica \\ Jeffry Chavarría Molina ${ }^{3}$ \\ Instituto Tecnológico de Costa Rica, Escuela de Matemática, \\ Cartago, Costa Rica
}

Recibido 14 de agosto de 2008 • Aceptado 29 de abril de 2009

\begin{abstract}
Resumen. PoliEstudio 1.0 es una herramienta computacional de licencia gratuita diseñada para el trabajo con polinomios en una variable y fue creado por un equipo del cual forman parte los autores de este artículo. Aquí se documenta la validación cualitativa realizada a este software, con el objetivo de brindar al Sistema Educativo Costarricense un programa educativo validado, que dé respuesta parcial a la problemática que aqueja la formación matemática de los estudiantes de secundaria, en particular, en los temas concernientes a polinomios en una variable y, específicamente, a los estudiantes de octavo año.
\end{abstract}

Palabras clave. Validación de software, herramienta computacional, enseñanza, polinomios.

Abstract. PoliEstudio 1.0 is a computational tool, with free license, created to work with polynomial expressions in one variable and it was created by a team in which the authors of this article are part of. This article documents the qualitative validation performed to this software which main objective was to bring to the Costa Rican Educational System a validated educational software that can solve, partially, the problems that nowadays exists in the mathematic education of secondary students, particularly in the topics related to polynomial expressions in one variable and specifically to those students who are in eighth grade.

Key words. Software validation, computational tool, teaching, polynomial expressions.

\footnotetext{
${ }^{1}$ Este informe corresponde al Trabajo Final de Graduación desarrollado por los autores para optar por el grado de Licenciados en Enseñanza de la Matemática en la Universidad Nacional.

${ }^{2}$ Bachiller en Enseñanza de la Matemática Asistida por Computadora del ITCR, Licenciado en Enseñanza de la Matemática de la UNA. Actualmente, cursa la Maestría en Matemática Aplicada de la UCR y labora como profesor de Matemática en la Escuela de Matemática del ITCR, desde el año 2005.

E-mail: jfallas@itcr.ac.cr

${ }^{3}$ Bachiller en Enseñanza de la Matemática Asistida por Computadora del ITCR, Licenciado en Enseñanza de la Matemática de la UNA. Actualmente, cursa la Maestría en Matemática Aplicada de la UCR y labora como profesor de Matemática en la Escuela de Matemática del ITCR, desde el año 2005.

E-mail: jchavarria@itcr.ac.cr
} 


\section{Introducción}

La educación matemática costarricense se caracteriza, actualmente, en muchos casos, por el uso de ciertas metodologías para la enseñanza, las que, aunque no podamos prescindir de ellas, es necesario complementarlas con otras que ayuden a enriquecer los procesos de enseñanza y de aprendizaje en el área de esta disciplina. La computadora y, en particular, los software educativos, se proyectan como una estrategia metodológica necesaria para la educación matemática en la actualidad.

La incorporación de herramientas tecnológicas en el aula de matemática, tales como un software especializado para un tema matemático determinado, le brinda al docente una opción novedosa para propiciar en sus estudiantes un mejor manejo de los conceptos, mediante procesos de exploración y de descubrimiento.

PoliEstudio 1.0 es un programa computacional elaborado en el segundo semestre del año 2004 por los autores de este artículo, junto con el Bachiller Pablo Acosta Rivera. Éste consiste en una herramienta creada, específicamente, para el trabajo con polinomios en una variable concebida como un complemento de una propuesta didáctica orientada a mejorar los procesos de enseñanza y de aprendizaje de polinomios en una variable.

Este escrito documenta la validación cualitativa realizada a PoliEstudio 1.0. Esta validación se consumó debido a la necesidad de que este recurso didáctico contara con un proceso de investigación que garantizara su pertinencia y calidad. El proyecto buscó brindar al Sistema educativo costarricense una herramienta computacional que diera respuesta parcial a la problemática que aqueja la formación matemática de los estudiantes de secundaria.

\section{Descripción del software}

En la Figura 1 se muestra la pantalla principal de PoliEstudio 1.0.

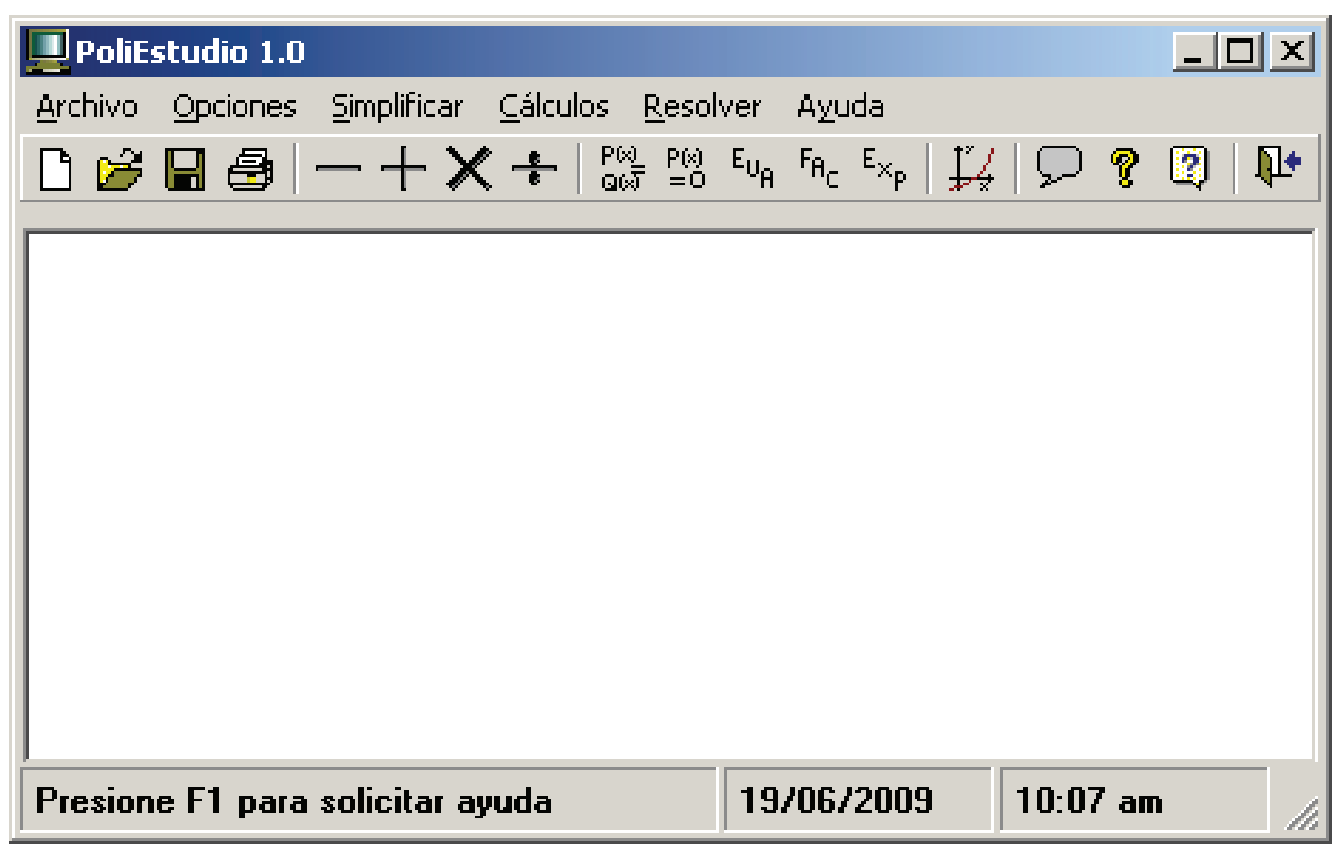

Figura 1. Pantalla principal de PoliEstudio 1.0. 
Las funciones que realiza el programa son la adición, la sustracción, la multiplicación y la factorización de polinomios. Además, resuelve ecuaciones polinomiales en el conjunto de los números reales y encuentra los ceros reales de un polinomio. De manera similar, realiza la división de polinomios y la división sintética, con la opción de mostrar en pantalla el procedimiento necesario.

La Figura 2 presenta el procedimiento mostrado por PoliEstudio cuando se utiliza la opción de División larga de polinomios.

\begin{tabular}{|c|c|c|c|c|c|}
\hline \multirow{3}{*}{$\begin{array}{r}x^{3} \\
-x^{3}\end{array}$} & & & & & $-x^{2}+1$ \\
\hline & $+-x^{2}$ & + & $0 \cdot x+$ & 1 & \multirow{5}{*}{$-x+1$} \\
\hline & $+0 \cdot x^{2}$ & + & $\mathbf{x}+$ & 0 & \\
\hline & $+-x^{2}$ & + & $\mathbf{x}+$ & 1 & \\
\hline & $+x^{2}$ & + & $0 \cdot x+$ & -1 & \\
\hline & & + & $x+$ & 0 & \\
\hline
\end{tabular}

Figura 2. División larga de polinomios.

La figura 3 muestra las opciones de Factorizar", "Expandir" y "Evaluación”.

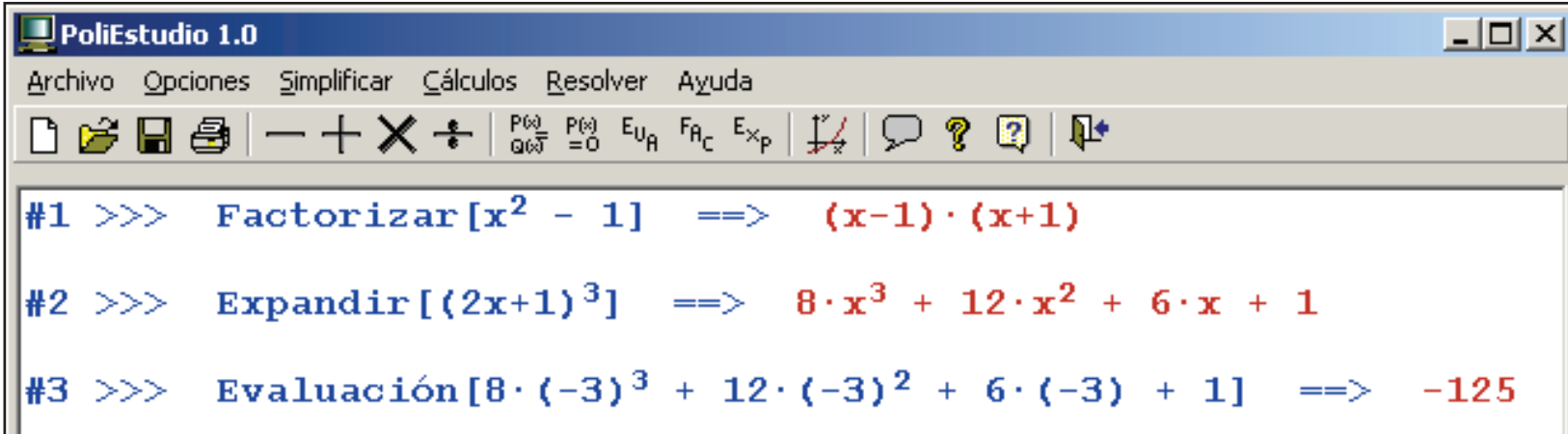

Figura 3. Opciones de Factorizar, Expandir y Evaluación.

Adicionalmente, PoliEstudio 1.0 cuenta con un graficador de funciones polinomiales que permite representar uno o varios polinomios en un sistema de coordenadas cartesianas. Esta aplicación se denomina el "PoliGraficador" y se muestra en la figura 4. 


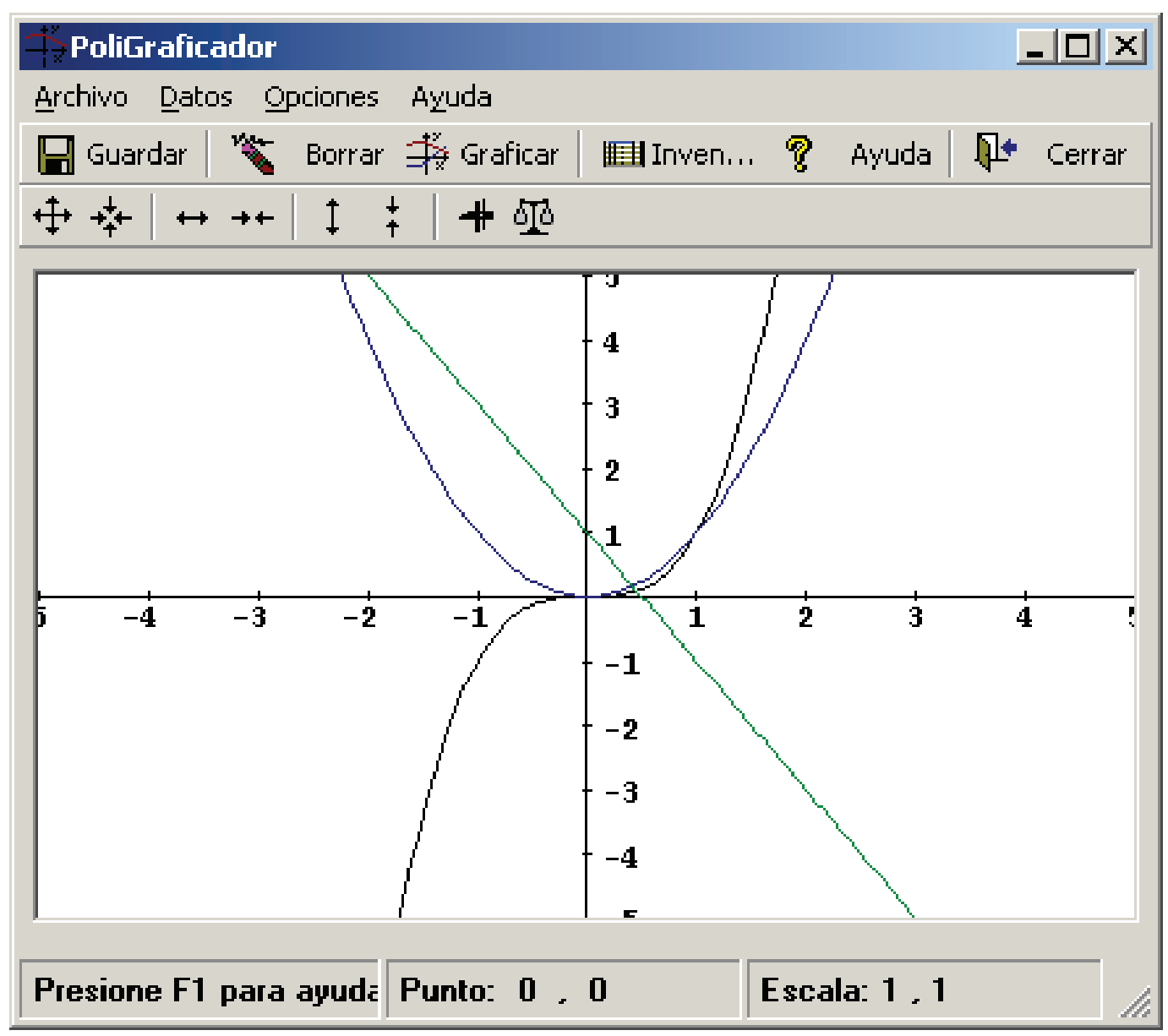

Figura 4. PoliGraficador.

Este programa cuenta con dos ayudas complementarias. Una de ellas explica cómo utilizar el programa y posee ejemplos e ilustraciones para indicar cada una de las funciones y operaciones que éste realiza. La otra contiene aspectos teóricos sobre polinomios en una variable, en la cual se encuentran definiciones, teoremas, resultados, procedimientos, algoritmos y ejercicios resueltos referentes a polinomios en una variable.

PoliEstudio 1.0 es un programa de licencia gratuita, por lo que profesores y estudiantes pueden utilizarlo sin ninguna restricción en el aula, en el laboratorio de informática o, si es preciso, en sus hogares.

\section{Pregunta y categorías de la investigación}

En la investigación desarrollada para la validación de PoliEstudio 1.0 se indagó a profundidad la siguiente pregunta.

¿Cuáles aspectos se deben fortalecer, modificar o incluir a la herramienta computacional PoliEstudio 1.0, para su mejoramiento como recurso didáctico para la enseñanza de temas de Álgebra, en la educación secundaria costarricense? 
No obstante, la investigación desarrollada también aportó información para aproximar una respuesta a la siguiente interrogante.

¿Qué características presenta la herramienta computacional PoliEstudio 1.0, que la identifican como un software educativo adecuado para contribuir con los procesos de enseñanza $y$ de aprendizaje de los temas relativos a polinomios en una variable, en el nivel de octavo año de secundaria?

Para la recolección de los datos se recurrió a las siguientes categorías de investigación.

1. Percepciones mostradas por los estudiantes y el profesor sobre la asignatura Matemática Asistida.

2. Dinámicas vividas en el grupo durante las clases en el laboratorio.

3. Actitudes mostradas por los estudiantes cuando trabajaron con las guías y con PoliEstudio 1.0 .

4. Metodología empleada para evaluar a los estudiantes en Matemática Asistida.

5. Aportes y recomendaciones realizadas por los participantes de la investigación sobre esta herramienta.

\section{Metodología de la investigación}

Con base en el propósito de la investigación, el paradigma cualitativo fue el que mejor se identificó con los objetivos y, por tanto, fue escogido para realizar el estudio. Se emplearon variadas técnicas para la recolección de datos, mediante las cuales se estudió un grupo de educandos en un contexto de enseñanza asistida por computadora. Se describieron las palabras y frases que emplearon los participantes de la manera más fiel a la forma en que las emitieron. Se usó la observación participante, la cual permitió observar las conductas de cada uno de ellos, desde una perspectiva holística.

Además, la validación realizada del software educativo PoliEstudio 1.0 se puede catalogar como un caso de investigación aplicada. Según Bunge y Sacristán (2000), en investigación científica "... si se persigue un fin puramente cognitivo, se obtiene ciencia pura; si la finalidad a largo plazo es utilitaria, resulta ciencia aplicada" (p. 23). Por tanto, una investigación se considera aplicada si su finalidad, a largo plazo, es generar un resultado práctico y útil que permita dar respuesta a un problema específico. Éste fue el caso de esta investigación.

Con respecto al método investigativo se adoptó, principalmente, el estudio de caso. Según Bermejo (2007), “... en el estudio de caso o monografía estudiamos sólo un objeto o un caso. El método también puede ser utilizado cuando se estudia un número de objetos con características similares" (parr. 1). Se partió del hecho de que los estudiantes del grupo con el que se llevó a cabo la investigación estaban cursando el mismo nivel y llevando las mismas materias con los mismos profesores, por lo que estos estudiantes contaron con características similares. Por este motivo, se adoptó este método de investigación con la variante de que el objeto de estudio fue un grupo de veinticinco personas.

\section{Espacio, temporalidad y participantes}

La investigación se desarrolló con un grupo de estudiantes de octavo año de un colegio privado localizado en la provincia de Cartago, Costa Rica. Esta institución tiene la particularidad 
de que es una de las pocas instituciones de enseñanza media en Costa Rica que incorpora dentro de sus planes de estudio la materia Matemática Asistida. Como parte de la oferta educativa de esta institución, a cada uno de los grupos, en todos los niveles, se les imparte semanalmente dos lecciones de Matemática Asistida de cuarenta minutos cada una. Estas lecciones se desarrollan en un laboratorio de computadoras debidamente equipado. En total, cada grupo recibe por semana seis lecciones de Matemática y dos lecciones de Matemática Asistida.

Además, la investigación se complementó con dos talleres de PoliEstudio 1.0. El primero de ellos se realizó con profesores de matemática de enseñanza media en el V Congreso Internacional sobre Enseñanza de la Matemática Asistida por Computadora (V CIEMAC). El segundo, con estudiantes avanzados de la carrera Bachillerato en Enseñanza de la Matemática Asistida por Computadora, del Instituto Tecnológico de Costa Rica.

El trabajo de campo de la investigación se desarrolló en un lapso de tres meses, a partir del mes de setiembre de 2007. La implementación de PoliEstudio 1.0 en el laboratorio se llevó a cabo en todas las lecciones efectivas de Matemática Asistida. Durante este período se realizaron observaciones participantes y se aplicaron las demás técnicas de recolección de información, a saber: recolección de documentos personales, aplicación de una prueba de diagnóstico y entrevistas. Adicionalmente, se complementó el estudio en un lapso de nueve meses, incluyendo análisis preliminar, la etapa final de análisis de datos, la programación de la versión 2.0 del software PoliEstudio y la redacción del informe final de la investigación.

\section{Resultados y conclusiones}

Hubo una gran variedad y riqueza en las sugerencias brindadas por los participantes de la investigación, en búsqueda del mejoramiento de PoliEstudio 1.0. Las sugerencias pueden agruparse de la siguiente manera:

1. La interfaz y la interactividad de PoliEstudio 1.0. Debían mejorarse aspectos como el diseño de los botones, íconos y ventanas, así como el uso de los colores. Además, debían agregarse opciones que hicieran el programa más interactivo. Por ejemplo, que el programa ofreciera opciones para que el usuario personalizara colores, tamaño y tipo de la fuente, entre otros.

2. El ingreso de los datos. Debía haber mayor flexibilidad para que el usuario ingresara los polinomios. El programa debía aceptar una mayor gama de expresiones polinomiales escritas de diversas formas.

3. El software de instalación. Debía cambiarse por un nuevo software de instalación, cuyas instrucciones estuvieran en español.

4. Los archivos de ayuda. Debían incluirse, en ambos archivos, más ejemplos. Además, se debía mejorar la redacción de los documentos, así como su calidad gráfica.

Se destaca como principal resultado de la investigación, el diseño de la segunda versión del programa, la cual incorpora la mayoría de las mejoras sugeridas por los participantes del proyecto. En la Figura 5 se muestra la pantalla principal de PoliEstudio 2.0. 


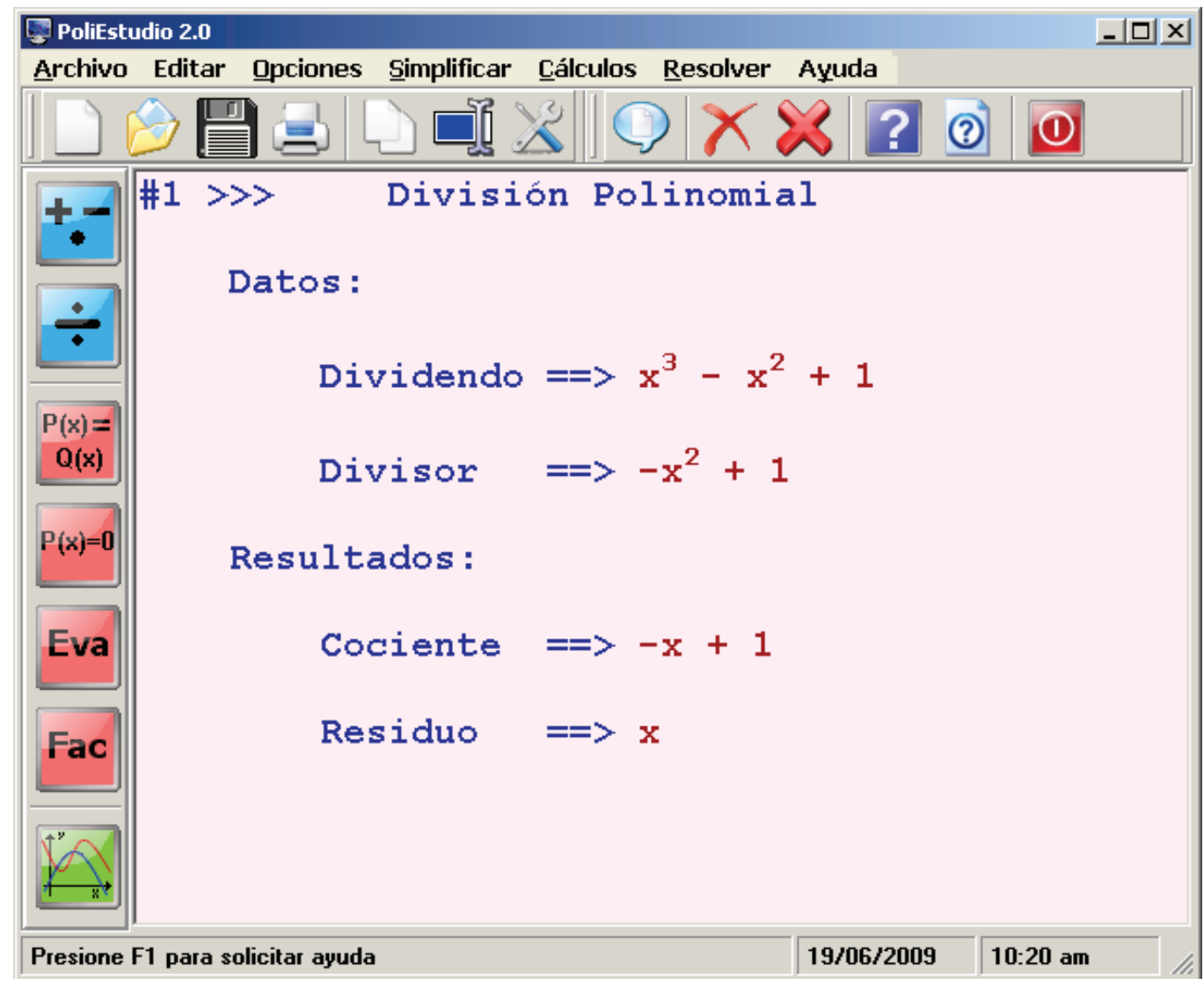

Figura 5. Pantalla principal de PoliEstudio 2.0.

Algunas de las novedades que incluye PoliEstudio 2.0 son:

1. Nuevos botones y mejor diseño gráfico de cada uno de ellos.

2. Una interfaz más agradable en todos los formularios y menús.

3. Opciones para cambiar el tipo, el color y el tamaño de la letra de la pantalla principal.

4. Mayor flexibilidad en cuanto al ingreso de los datos, ya que se implementó un nuevo algoritmo para que el programa permitiera expresiones polinomiales, sin importar las formas equivalentes en que el usuario las ingrese.

5. Reelaboración de los archivos de ayuda e incorporación de nuevos ejemplos.

6. Mejor calidad en ambos archivos de ayuda.

7. Software de instalación en español.

\section{Conclusiones}

Con base en el proceso de investigación vivido, se pudieron cristalizar, entre otras, las siguientes conclusiones:

- Todo software diseñado con fines educativos, ya sea con la intención de comercializarlo o no, debe ser sometido a etapas de validación. Debe ser analizado tanto por personas con experiencia en software educativo, como por potenciales usuarios. 
- De acuerdo con la edad de los estudiantes y el nivel que cursan, las demandas y preferencias sobre un programa educativo son distintas. En la investigación desarrollada, la calidad gráfica de PoliEstudio 1.0 fue el aspecto en el que más se recibieron recomendaciones.

- Como no siempre se tiene garantía sobre la calidad del software educativo que se tiene a disposición, es importante que el docente analice cualquier programa antes de emplearlo en su clase, principalmente, en los casos de software libre y software gratuito.

- $\quad$ Todo software educativo debe contar con un archivo de ayuda lo más completo posible. Idealmente, se debe incorporar algún archivo que contenga teoría y ejemplos del tema sobre el cual trata el programa. Un programa es mucho más completo y didáctico si el estudiante tiene la posibilidad de consultar la teoría en él.

\section{Recomendaciones}

- $\quad$ En términos generales, se lograron los objetivos planteados al iniciar el estudio; sin embargo, el tema de investigación seleccionado da cabida a futuros estudios que pueden complementar y fortalecer los resultados obtenidos en el nuestro.

- Recalcamos que el enfoque de la investigación desarrollada se centró en la validación del software educativo PoliEstudio 1.0. Para ello, se aplicó esta herramienta computacional a un grupo de octavo año del tercer ciclo. A causa de las características particulares de este grupo y a nuestro interés, la investigación se enfocó hacia un repaso de ciertos conceptos matemáticos apoyados en PoliEstudio 1.0. Además, se aprovechó el conocimiento que los estudiantes fueron obteniendo sobre el software durante el proceso, para recoger sugerencias y recomendaciones que permitieran mejorar la calidad del software en cuestión. La validación realizada buscó mejorar la calidad técnica, el diseño y los aspectos funcionales del programa.

- Por tanto, es importante considerar, como una línea futura de investigación, realizar una validación de PoliEstudio 2.0, pero buscando analizarlo como una herramienta didáctico-pedagógica, que potencie el aprendizaje de los estudiantes. Sería bueno analizar los aportes que este software puede ofrecer al momento de enseñar los conceptos y no sólo como una herramienta de repaso.

- $\quad$ Finalmente, se recomienda el uso del software PoliEstudio 2.0 como una herramienta de apoyo al docente para la enseñanza de los temas de Álgebra: operaciones con polinomios (incluyendo división de polinomios y división sintética), ecuaciones polinomiales y factorización de polinomios en una variable, así como en el tema de funciones polinomiales. La validación se realizó en el nivel de octavo año; sin embargo, las bondades de este software se podrían explotar en otros niveles y contextos.

\section{Referencias bibliográficas}

Bermejo, J. (2007). Estudio de Caso. Recuperado el 15 de marzo de 2007, de http://www2.uiah.fi/ projects/metodi/271.htm

Bunge, M. \& Sacristán, M. (2000). La investigación científica: su estrategia y su filosofía. Madrid, España: Siglo XXI. 


\section{Otras fuentes consultadas}

Cataldi, Z., Lage, F., Pessacq, R. \& García, R. (1999). Ingeniería de software educativo. Recuperado el 8 de octubre de 2007, de

http://www.fi.uba.ar/laboratorios/lsi/c-icie99-ingenieriasoftwareeducativo.pdf

Cataldi, Z. (2000). Metodología de diseño, desarrollo y evaluación de software educativo. Tesis de Maestría, Facultad de Informática, Universidad Nacional de la Plata, Argentina.

Chavarría, J. \& Fallas, J. (2008). Validación del software educativo PoliEstudio 1.0. Tesis de Licenciatura, Escuela de Matemáticas, Universidad Nacional, Costa Rica.

Colás, M. P. \& Buendía, L. (1998). Investigación educativa. Sevilla, España: Alfar.

González R., B. (2002). Criterios y métodos de evaluación de software educativo. Recuperado el 29 de setiembre de 2007, de http://byrong.iespana.es/public/evsoftwared.pdf

Marqués, P. (1996). El software educativo. Universidad Autónoma de Barcelona. Recuperado el 5 de setiembre de 2007, de http://www.Imi.ub.es/te/any96/marques_software/\#index

Marqués, P. (1998). Programas didácticos: diseño y evaluación. Recuperado el 15 de setiembre de 2007, de http://www.karisma.org.co

Taylor, S. y Bogdan, R. (1996). Introducción a los métodos cualitativos de investigación: La búsqueda de significados. ( $3^{\mathrm{a}}$ Reimpr.). Madrid, España: Paidós. 